\title{
Silicon photonics for multicore fiber communication
}

Ding, Yunhong; Kamchevska, Valerija; Dalgaard, Kjeld; Bacco, Davide; Rottwitt, Karsten; Hu, Hao; Galili, Michael; Morioka, Toshio; Oxenløwe, Leif Katsuo

\section{Published in:}

Proceedings of 2016 Asia Communications and Photonics Conferene

Link to article, DOI:

10.1364/ACPC.2016.AF1G.1

Publication date:

2016

Document Version

Peer reviewed version

Link back to DTU Orbit

Citation (APA):

Ding, Y., Kamchevska, V., Dalgaard, K., Bacco, D., Rottwitt, K., Hu, H., Galili, M., Morioka, T., \& Oxenløwe, L. K. (2016). Silicon photonics for multicore fiber communication. In Proceedings of 2016 Asia Communications and Photonics Conferene [Paper AF1G.1] Optical Society of America (OSA). https://doi.org/10.1364/ACPC.2016.AF1G.1

\section{General rights}

Copyright and moral rights for the publications made accessible in the public portal are retained by the authors and/or other copyright owners and it is a condition of accessing publications that users recognise and abide by the legal requirements associated with these rights.

- Users may download and print one copy of any publication from the public portal for the purpose of private study or research.

- You may not further distribute the material or use it for any profit-making activity or commercial gain

- You may freely distribute the URL identifying the publication in the public portal 


\title{
Silicon photonics for multicore fiber communication
}

\author{
Yunhong Ding*, Valerija Kamchevska, Kjeld Dalgaard, Davide Bacco, Karsten Rottwitt, \\ Hao Hu, Michael Galili, Toshio Morioka, and Leif Katsuo Oxenløwe \\ Technical University of Denmark, Department of Photonics Engineering, Kongens Lyngby, Denmark \\ yudin@fotonik.dtu.dk
}

\begin{abstract}
We review our recent work on silicon photonics for multicore fiber communication, including multicore fiber fan-in/fan-out, multicore fiber switches towards reconfigurable optical add/drop multiplexers. We also present multicore fiber based quantum communication using silicon devices.
\end{abstract}

OCIS codes: (230.3120) Integrated optics devices; (060.2330) Fiber optics communications; (270.5568) Quantum cryptography

\section{Introduction}

Nowadays, driven by the huge capacity demand, the communication capacity over standard single mode fibers is approaching its theoretical limitation [1]. Space division multiplexing (SDM) using multicore fibers (MCFs) has shown to be a promising technology to further increase the communication capacity over a single fiber [2-4]. To achieve a real commercial success, SDM must also be compatible with optical network implementations. In particular, it should support optical routing at reconfigurable optical add/drop multiplexer (ROADM) nodes in future MCF networks, as illustrated in Fig. 1. The crucial devices are ultra-compact, high efficient MCF fan-in/fan-out (FI/FO) devices [5-8], core switches of MCFs and MCF switches [9-13].

On the other hand, in quantum communication community, similarly to classical communication, SDM can also be an efficient means to increase the information efficiency (bit/photon). So far quantum key distribution (QKD) protocols over free-space using optical angular momentum modes have been reported [14-16].

In this paper, we review our experimental results on silicon photonics for MCF communication, including crucial devices of MCF couplers, MCF switches towards MCF ROADM. In addition, we present novel QKD protocol based on MCF using silicon chips.

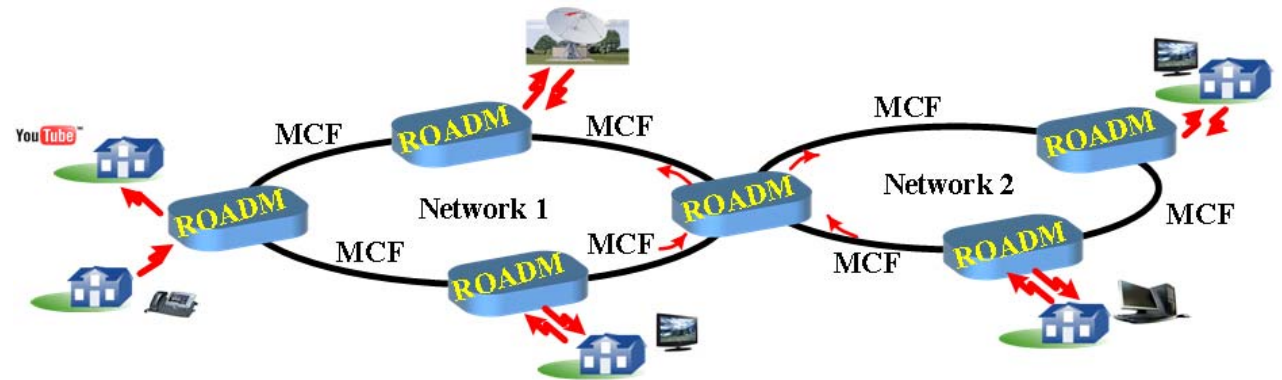

Fig. 1. A conceptual MCF network. The introduction of ROADMs enables users to drop data from and add data to the network, as well as to communicate between networks.

\section{MCF FI/FO}

MCF FI/FO is one of the most important devices for MCF communication. Free space based couplers [5] is an efficient solution providing high coupling efficiencies with large bandwidths and polarization independence. However, they are usually very bulky. A more compact solution by physical-contact type FI/FOs [6] has been reported. From an integration point of view, on-chip MCF FI/FOs is preferred so that many FI/FOs and other functionalities can be integrated on the same chip, making compact chip-based MCF ROADM possible. Three dimensional (3D) waveguides fabricated by ultrafast laser inscription [7] have been reported. Grating coupler based MCF FI/FO is another efficient solution [8]. As shown in Fig. 2, the layout of the output grating couplers corresponds to that of the cores of the MCF with the same pitch as the MCF. The on-chip 7-core fiber FI/FO was fabricated on the silicon-oninsulator (SOI) platform with bottom aluminum (Al) mirror that was introduced by flip-bonding method [13]. The grating couplers are fully-etched [17] so that they can be simultaneously fabricated with silicon wires in a single lithography and etching step. A highest coupling efficiency of $-3.8 \mathrm{~dB}$ with $3 \mathrm{~dB}$ coupling bandwidth of $48 \mathrm{~nm}$ and $1.5 \mathrm{~dB}$ bandwidth covering the whole $\mathrm{C}$ band were achieved. At the same time, low coupling efficiency variation of $1.5 \mathrm{~dB}$ between all spatial channels with crosstalk below $-32 \mathrm{~dB}$ were demonstrated. 

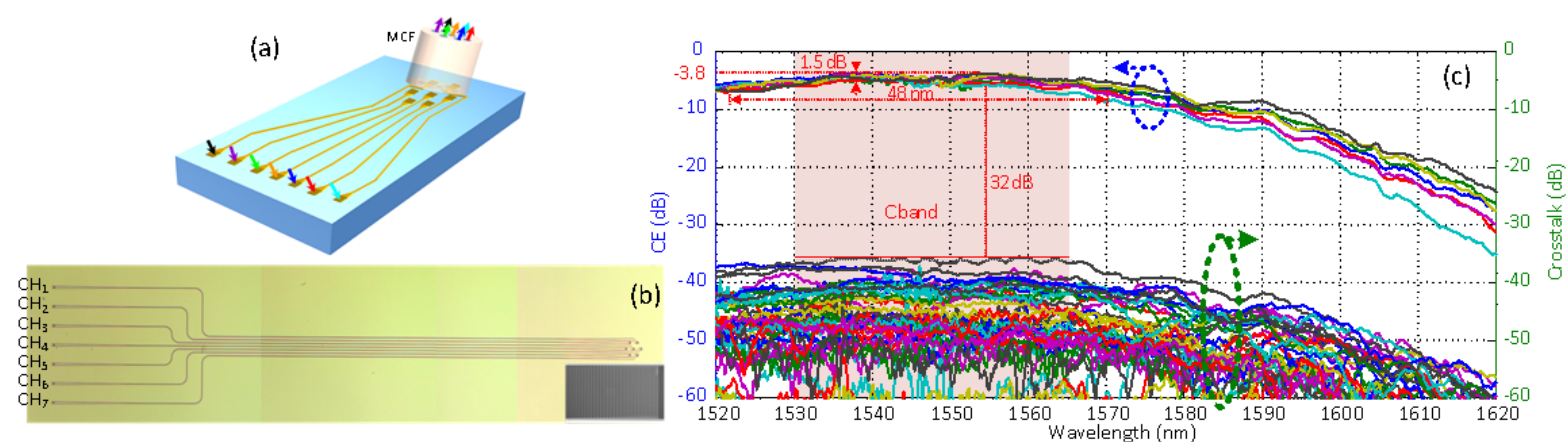

Fig. 2. (a) Schematic of the grating coupler-based MCF FI/FO. (b) Fabricated MCF FI/FI. (c) Measured coupling efficiency and crosstalk for the MCF FI/FO.

\section{On-chip MCF switches towards MCF ROADM}

Core switches and MCF switches are basic functionalities for MCF ROADM module. A ROADM for MCFs communication systems has been proposed using an array of wavelength-selective switches (WSSs) [9]. Micro Electro Mechanical Systems (MEMS) mirrors or on Liquid Crystal on Silicon (LCOS) pixel arrays have also been reported for MCF switching [9, 10]. All-optical nonlinear switching in MCFs has reported using high-power ultrashort laser pulses [11]. These solutions tend to have large insertion loss. Flexural acoustic waves have been used for switching in MCFs with low insertion loss [12]. Thanks to the grating coupler array based MCF FI/FO, we achieve core switching of MCFs on an SOI platform. The topological scheme is shown in Fig. 3(a), which consists of MCF couplers and $7 \times 7$ switching matrix [13]. A seven-core fiber is coupled to the input MCF coupler. After switching, the seven spatial channels are coupled to the output MCF through a second MCF coupler. In this scheme, any core of the input MCF can be reconfigurably routed to any core of the output MCF fiber. As shown in Fig. 3(a), by configuring the corresponding MZIs, the bar (solid line) switching configuration for the seven spatial channels (corresponding to different color) can be reconfigured to the cross (dash line) configuration. Fig. 3(b) shows the fabricated silicon chip, where the $7 \times 7$ switch consists of 57 Mach-Zehnder interferometers (MZIs), each incorporating a heater in one arm. Fig. 3(c) shows the transmission and corresponding crosstalk for bar configuration (core 1 input for core 1 at the output, core 2 to core 2, etc.). High transmission covering the whole C-band is obtained for all the switching paths with crosstalk lower than $-30 \mathrm{~dB}$. A lowest insertion loss of $4.5 \mathrm{~dB}$ with channel dependent loss lower than $2.5 \mathrm{~dB}$ is achieved. By applying proper voltages to the corresponding heaters in the $7 \times 7$ switching matrix, the silicon PIC is tuned to cross switching configuration (core 1 input for core 7 at the output, core 2 to core 6 , etc.). High transmission is still obtained for all switching paths with lowest insertion loss of $5.5 \mathrm{~dB}$ and $2.2 \mathrm{~dB}$ channel dependent loss. Low crosstalk (lower than $-35 \mathrm{~dB}$ ) is obtained for the whole C-band.

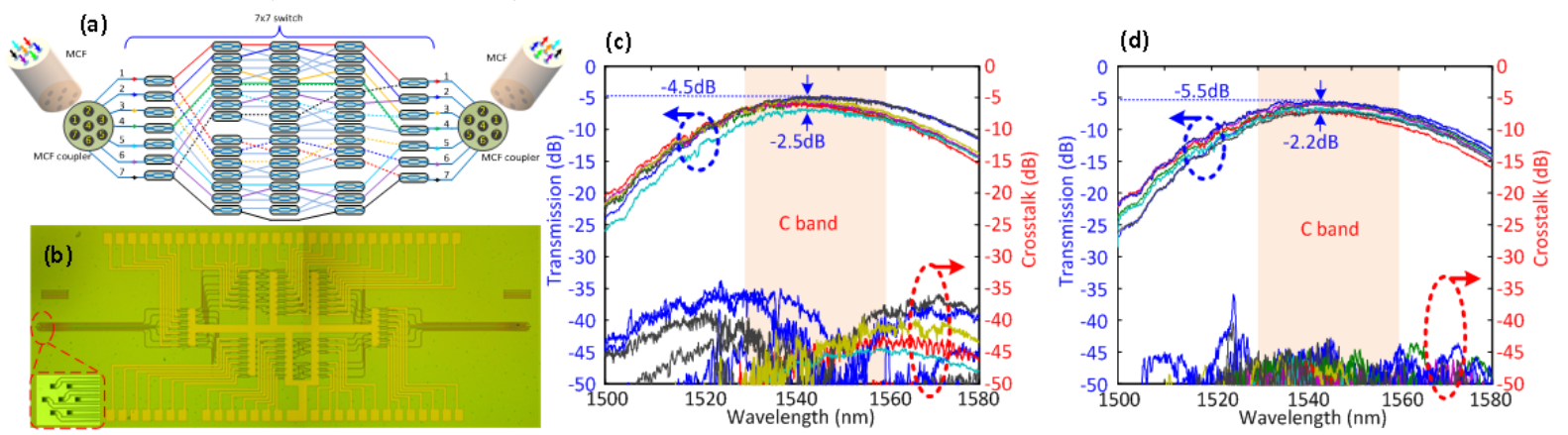

Fig. 3. (a) Topological scheme of the silicon PIC for core switch of MCFs. (b) Fabricated device. The inset shows the grating coupler array based MCF FI/FO. Measured transmission and corresponding crosstalk for (c) bar and (d) cross switching configurations.

\section{MCF based QKD}

Recently, the quantum community has put tremendous effort on increasing information efficiency for QKD. Usually the traditional QKD schemes are binary system, where the information efficiency is intrinsically limited to 1 bit/photon. SDM is an efficient means to increase the information efficiency. OAM modes are used to realize highdimensional QKD protocols over free-space link [13-15]. MCF can also been utilized for QKD application. One advantage of MCFs is the crosstalk between each cores is very small, making superposition of quantum states in different cores of MCF possible. Fig. 4 shows the experimental setup of using MCF for 2 independent-BB84 
protocol. Instead of using polarization domain to encode the information in traditional BB84 protocol, we use spatial (core) dimension in MCF to create two independent mutually unbiased basis, i.e. A/B base $\{|\mathrm{A}\rangle,|\mathrm{B}\rangle ;|\mathrm{A}\rangle+|\mathrm{B}\rangle,|\mathrm{A}\rangle-|\mathrm{B}\rangle\}$ and $\mathrm{C} / \mathrm{D}$ base $\{|\mathrm{C}\rangle,|\mathrm{D}\rangle ;|\mathrm{C}\rangle+|\mathrm{D}\rangle,|\mathrm{C}\rangle-|\mathrm{D}\rangle\}$. Here, $|\mathrm{A}\rangle,|\mathrm{B}\rangle,|\mathrm{C}\rangle$ and $|\mathrm{D}\rangle$ are the quantum states related to the four individual cores. Each base implements an independent BB84 protocol. Tomography measurement is carried out under the condition of weak coherent pulses $(\mu<0.2)$ of the four basis, which are also shown in Fig. 4. Good agreement between theoretical and experimental result is achieved, indicating that the two independent bases are well prepared.

(a)

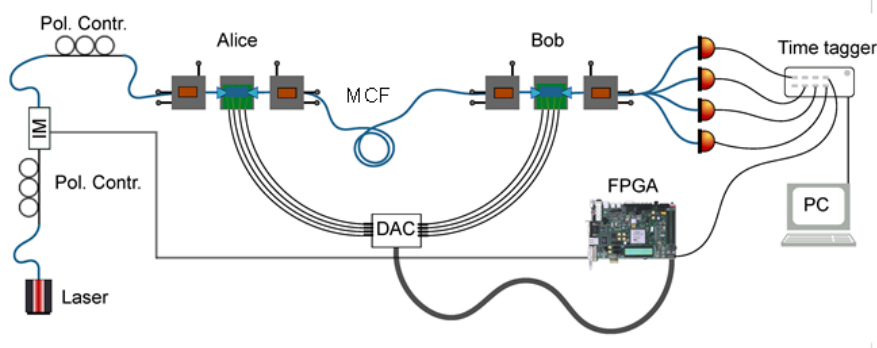

(b)

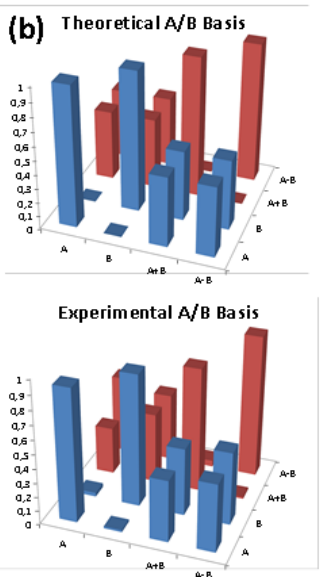

(c) Theoretical C/D Basis

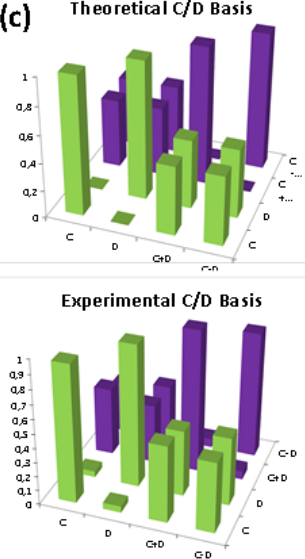

Fig. 4. (a) Experimental setup of 2 independent BB84 protocol using MCF. Theoretical analysis of quantum tomography and measured results of (b) A/B and (c) C/D base.

\section{Conclusion}

We have investigated silicon integrated devices for MCF communication, including high-efficiency MCF FI/FO based on grating coupler array, core switches of MCFs. We also present a novel MCF based QKD protocol using silicon photonic integrated circuits.

The work is supported by projects DFF-1337-00152, DFF-1335-00771, and SPOC research centre of excellence (ref DNRF123).

\section{References}

[1]. R.-J. Essiambre, et al. "Capacity limits of optical fiber networks,” J. Lightwave Technol. 28, 662-701 (2010).

[2]. H. Takara et al., "1.01-Pb/s (12 SDM/222 WDM/456 Gb/s) crosstalk-managed transmission with 91.4-b/s/Hz aggregate spectral efficiency," in ECOC'2012, paper Th.3.C.1.

[3]. H. Hu et al. "1.28 Tbaud Nyquist-OTDM transmission over a 7-Core fiber using an on-chip SDM coupler," in OECC'2014, paper THPDP2-2.

[4]. T. Mizuno et al., "12-core×3-mode dense space division multiplexed transmission over 40 km employing multi-carrier signals with parallel MIMO equalization," in OFC/NFOEC'2014, paper Th5B.2.

[5]. J. Sakaguchi et al., "19-core fiber transmission of 19×100×172-Gb/s SDMWDM- PDM-QPSK signals at 305 Tb/s," in OFC/NFOEC'2012, paper PDP5C.1.

[6]. Y. Abe et al., "Low-loss physical-contact-type fan-out device for 12-core multicore fiber," in ECOC'2013, paper P.1.7.

[7]. R. R. Thomson et al., "Ultrafast laser inscription of a 121-waveguide fan-out for astrophotonics," Opt. Lett. 37, 2331-2333 (2012).

[8]. Y. Ding et al., "On-chip grating coupler array on the SOI platform for fan-in/fan-out of MCFs with low insertion loss and crosstalk," Opt. Express, 23, 3292-3298 (2015).

[9]. R. Ryf, "SDM components and MIMO experiments in multimode and multicore fibers," in ECOC'2014, paper Th.2.1.1.

[10]. L. Nelson et al., "Spatial superchannel routing in a two-span ROADM system for space division multiplexing," J. Lightwave Technol. 32, 783-789 (2014).

[11]. M. Liu and K. S. Chiang, "Nonlinear switching of ultrashort pulses in multicore fibers," IEEE J. Sel. Top. Quantum Electron. 47, 14991505 (2011).

[12]. G. M. Fernandes et al., "Switching in multicore fibers using flexural acoustic waves," Opt. Express 23, 26313-26325 (2015).

[13]. Y. Ding et al., "Reconfigurable SDM switching using novel silicon photonic integrated circuit," arXiv:1608.05645, 2016.

[14]. M. Mirhosseini et al., "High-dimensional quantum cryptography with twisted light," New J. Phys. 17, 033033 (2015).

[15]. R. W. Boyd et al., "Quantum key distribution in a high-dimensional state space: exploiting the transverse degree of freedom of the photon," Proc. SPIE 7948, 79480L (2011).

[16]. M. Mafu et al., "Higher-dimensional orbital-angular-momentum-based quantum key distribution with mutually unbiased bases," Phys. Rev. A 88, 032305 (2013).

[17]. Y. Ding, H. Ou, and C. Peucheret, "Ultrahigh-efficiency apodized grating coupler using fully etched photonic crystals," Opt. Lett. 38, 2732-2734 (2013). 